\title{
Preparation Technique Affects Recipient Immune Targeting of Autologous Mesenchymal Stem Cells
}

OPEN ACCESS

Edited by:

Debbie Guest,

Royal Veterinary College (RVC), United Kingdom

Reviewed by:

Jayesh Dudhia,

Royal Veterinary College (RVC),

United Kingdom

Janina Burk,

University of Giessen, Germany

*Correspondence:

Ashlee E. Watts

awatts@cvm.tamu.edu

Specialty section:

This article was submitted to Veterinary Regenerative Medicine,

a section of the journal

Frontiers in Veterinary Science

Received: 11 June 2021

Accepted: 20 August 2021

Published: 14 September 2021

Citation:

Rowland AL, Burns ME, Levine GJ and Watts AE (2021) Preparation Technique Affects Recipient Immune Targeting of Autologous Mesenchymal Stem Cells. Front. Vet. Sci. 8:724041. doi: 10.3389/fvets.2021.724041

\author{
Aileen L. Rowland ${ }^{1}$, Madison E. Burns ${ }^{1}$, Gwendolyn J. Levine ${ }^{2}$ and Ashlee E. Watts ${ }^{1 *}$ \\ ${ }^{1}$ Department of Large Animal Clinical Sciences, Texas A\&M University, College Station, TX, United States, ${ }^{2}$ Department of \\ Veterinary Pathobiology, Texas A\&M University, College Station, TX, United States
}

Fetal bovine serum (FBS) is used for MSC preparation in pre-clinical animal models and veterinary applications, recently in US clinical trials, and for MSC products with current foreign market authorizations. The effect of anti-bovine titers, which are common in animals and humans, has not been investigated. In the equine model, where anti-bovine titers are universally high due to routine vaccination, we evaluated the recipient immune response to autologous MSCs prepared with and without FBS. Preparation of MSCs with FBS resulted in post injection inflammation and antibody mediated cytotoxicity of MSCs when compared to MSCs prepared without FBS. Importantly, synovial MSC concentrations were reduced and LPS induced pain was higher, when FBS was used to prepare MSCs, demonstrating reduced efficacy of FBS prepared MSCs. Fetal bovine serum should no longer be utilized for MSC preparation in pre-clinical study, clinical study, or veterinary applications. The use of FBS in previously reported studies, and in MSC therapeutics with current foreign market authorization, should be considered when interpreting results.

Keywords: mesenchymal stem cell, fetal bovine serum, bone marrow supernatant, horse, intra-articular, immunogenicity, equine, mesenchymal stromal cell

\section{INTRODUCTION}

Despite decades of work, consistent and reproducible clinical efficacy of mesenchymal stem cells (MSCs) has not been demonstrated (1-3). Failure to meet clinical endpoints in both late phase clinical trials and post-approval monitoring has precluded market authorization in the United States (1-4). Likewise, lack of predictable efficacy of non-human MSCs plagues the veterinary community and has casted doubt on the usefulness of MSCs, both translationally and clinically. One reason for the lack of consistent efficacy may be MSC preparation technique (1). Pittenger et al. recently emphasized the importance of MSC preparation technique, stating that the preparation method of MSCs is the product (5). Preparation methods include culture media composition and serum supplement sources.

Supplementation of culture media with fetal bovine serum (FBS) has been a standard MSC preparation technique since MSCs were first described in the 1970s, providing growth factors, hormones, and other undefined, yet essential, components to cell culture media (6). However, the use of FBS is decreasing because of ethical concerns, availability, and the risk of disease transmission from bovine products (7). Despite this shift in FBS acceptance, FBS supplemented MSCs have market approval for use in humans in Canada and New Zealand, and FBS supplementation remains the industry standard in pre-clinical and veterinary MSC use (4, 5, 8-11). 
An important, but infrequently discussed, consequence of FBS supplementation during MSC preparation is the accumulation of intracellular bovine contamination that is presented on MHCI, which leads to seroconversion of the recipient (12-15). In horses, we confirmed that the accumulation of intracellular bovine proteins by MSCs leads to local inflammation after therapeutic administration, but did not assess anti-bovine titers (16). In that report, removal of FBS during the final $48 \mathrm{~h}$ of culture markedly reduced intracellular bovine contamination, but all MSCs remained positive for intracellular bovine proteins (16).

A lack of change in anti-bovine titers in horses and cats after MSC therapy has led others to conclude that FBS contamination is not clinically relevant yet, in humans there is evidence that seroconversion against bovine proteins in MSC recipients correlates to poor clinical response $(11,17,18)$. The question remains, what do pre- and post-MSC treatment antibovine titers mean in patients receiving FBS supplemented MSCs? Immune recognition of intracellular bovine proteins and resultant cytotoxicity could explain why pre-clinical study often fails to predict therapeutic response and why human clinical trials have failed to meet rigorous clinical endpoints in the United States $(2,5,9,19)$.

Our objective was to determine if there is an immune response against autologous MSCs because of laboratory preparation with FBS. First, we confirmed that replacement of FBS supplementation with bone marrow supernatant (BMS) supplementation did not alter MSC growth or characterization. In the equine model, we then performed repeated intra-articular injections of autologous FBS supplemented MSCs (FBS-MSCs) or autologous BMS supplemented MSCs (BMS-MSCs). We demonstrate immune recognition with antibody mediated death of MSCs, local inflammation, and reduced efficacy after FBSMSC administration, which did not occur with BMS-MSCs. Given the historical and ongoing use of FBS in pre-clinical and clinical trials, identifying FBS use and potential recipient immune recognition with subsequent antibody mediated MSC death is imperative in interpreting results. In future study, especially pre-clinical study and veterinary applications where FBS supplementation remains the standard practice, FBS should not be utilized.

\section{MATERIALS AND METHODS}

\section{Animals and Experimental Overview}

All animals were cared for according to university standards and all procedures were approved by the animal care and use committee (AUP 2018-0003 and 2018-0118). Six horses were utilized for in vitro experiments (BMS characterization; five females, one castrated male), and 18 horses were utilized for the in vivo experiments (fetlock model; 13 females, five castrated males). All horses were Quarter Horse type and ranged from 4 to 22 years of age (median, 13; Table 1). For the in vivo experiment, the left metacarpophalangeal joint was utilized, and all joints were healthy with no known joint pathology. Horses were randomly assigned to groups by drawing group assignment from a hat (FBS, autologous BMS, pooled BMS).
TABLE 1 | Age, and gender ( $F=$ female; $G$ = gelding, castrated male) of horses in both the in vitro and in vivo experiments.

\begin{tabular}{|c|c|c|c|}
\hline & Group Assignment & Age & Gender \\
\hline \multicolumn{4}{|l|}{ In vitro } \\
\hline Horse 1 & In vitro & 14 & $\mathrm{~F}$ \\
\hline Horse 2 & In vitro & 16 & $\mathrm{~F}$ \\
\hline Horse 3 & In vitro & 8 & $\mathrm{~F}$ \\
\hline Horse 4 & In vitro & 12 & $\mathrm{~F}$ \\
\hline Horse 5 & In vitro & 9 & $\mathrm{~F}$ \\
\hline Horse 6 & In vitro & 4 & $\mathrm{~F}$ \\
\hline \multicolumn{4}{|l|}{ In vivo } \\
\hline Horse 1 & FBS & 18 & $\mathrm{~F}$ \\
\hline Horse 2 & Autologous BMS & 16 & $\mathrm{~F}$ \\
\hline Horse 3 & Autologous BMS & 12 & $\mathrm{~F}$ \\
\hline Horse 4 & Pooled BMS & 12 & G \\
\hline Horse 5 & FBS & 22 & G \\
\hline Horse 6 & Autologous BMS & 4 & $G$ \\
\hline Horse 7 & Pooled BMS & 13 & $\mathrm{~F}$ \\
\hline Horse 8 & Pooled BMS & 14 & $\mathrm{~F}$ \\
\hline Horse 9 & FBS & 12 & $\mathrm{~F}$ \\
\hline Horse 10 & Pooled BMS & 11 & $\mathrm{~F}$ \\
\hline Horse 11 & Autologous BMS & 12 & $G$ \\
\hline Horse 12 & Autologous BMS & 12 & $\mathrm{~F}$ \\
\hline Horse 13 & FBS & 13 & $\mathrm{~F}$ \\
\hline Horse 14 & Pooled BMS & 13 & $\mathrm{~F}$ \\
\hline Horse 15 & FBS & 12 & $\mathrm{~F}$ \\
\hline Horse 16 & Pooled BMS & 11 & $\mathrm{~F}$ \\
\hline Horse 17 & Autologous BMS & 19 & G \\
\hline Horse 18 & FBS & 18 & $\mathrm{~F}$ \\
\hline
\end{tabular}

All MSCs were autologous.

Bone marrow obtained from six horses was portioned to two groups for MSC isolation and expansion in media supplemented with autologous BMS (one group, $n=6$ ) or FBS (second group, $n=6$ ). In vitro growth rate, characterization, and immunomodulation were compared in a paired analysis.

For in vivo evaluation, MSCs were isolated and expanded in autologous $(n=6)$ or pooled $(n=6)$ BMS, or FBS $(n=6)$ and cryopreserved in recipient serum. Intra-articular injection of autologous FBS-prepared MSCs $(n=6)$ or autologous BMSprepared MSCs (pooled BMS, $n=6$; or autologous BMS, $n$ $=6$ ) was performed on days 0 and 29 . When no differences in clinical assessments were detected between groups when MSCs were prepared with autologous or pooled BMS, data were combined (BMS and pooled) for FBS $(n=6)$ vs. BMS $(n=12)$ testing. To mimic naturally occurring inflammation, aqueous lipopolysaccharide (LPS) was injected immediately prior to MSCs at the second intra-articular injection. Clinical reaction, synovial cytology, synovial cytokines, synovial MSC concentration and cytotoxicity against MSCs were evaluated for the week after each intra-articular injection.

\section{MSC Isolation and Expansion}

Bone marrow was collected from the sternum as previously described (20). For the in vitro portion, $\sim 120 \mathrm{mls}$ of bone 
marrow was collected. For the in vivo portion, $\sim 360 \mathrm{mls}$ of bone marrow was collected. Heparinized bone marrow was centrifuged at $300 \mathrm{~g}$ for $5 \mathrm{~min}$ and the BMS was collected and filtered. The cellular fraction underwent red blood cell lysis as previously described (21). Briefly, red blood cell lysing solution $(7.7 \mathrm{mg} / \mathrm{ml} \mathrm{NH4CL;} 2.06 \mathrm{mg} / \mathrm{ml}$ hydroxymethaneaminomethane, $\mathrm{pH}$ 7.2) was added to the cellular fraction and centrifuged twice ( $300 \mathrm{~g}$ for $5 \mathrm{~min}$ ). The cells were washed in DPBS (Dulbecco's phosphate buffered saline, Corning) and resuspended in serum free media [Dulbecco's modified Eagle's medium (DMEM, Corning), $1 \mathrm{~g} / \mathrm{l}$ glucose supplemented with $10,000 \mathrm{U} / \mathrm{ml}$ Penicillin; $10 \mathrm{mg}$ streptomycin sulfate, $25 \mu \mathrm{g} / \mathrm{ml}$ amphotericin B (Gibco); 2.5\% HEPES buffer (Life Technologies); $10 \mu \mathrm{g} / \mathrm{ml}$ human recombinant basic fibroblastic growth factor (bFGF, Corning)]. Media contained either 10\% BMS (autologous or pooled) or $10 \%$ FBS (HyClone) and MSCs were maintained at $37^{\circ} \mathrm{C}$ in $5 \% \mathrm{CO}_{2}$, humidified air with media that was replaced with fresh media 3 times per week. Pooled BMS was created with equal parts from each of the six horses in the autologous BMS group.

After 7 days in culture, MSCs were passaged and replated at 5,000-7,000 MSCs/ $\mathrm{cm}^{2}$, which was repeated each time confluence reached $70-80 \%$ until the third passage. Once MSCs reach the third passage (P3), they were cryopreserved is cryopreservation media (95\% autologous serum and 5\% DMSO) as previously described (21).

\section{Characterization of BMS-MSCs and FBS-MSCs}

\section{In Vitro Colony Forming Unit-Fibroblast Assay}

The equivalent of $1 \mathrm{ml}$ of raw bone marrow was plated to $10 \mathrm{~cm}$ tissue culture dishes and maintained in media supplemented with $10 \%$ BMS or $10 \%$ FBS. After 10 days, colonies were stained with $3 \%$ crystal violet (Sigma Aldrich) and counted.

\section{Population Doubling Time}

Population doubling time was calculated using the following equation: $\mathrm{PDT}=$ days in culture ${ }^{*} \log 2 /(\log f-\log i)$ where $f$ is final cell count and $i$ is the initial number of cells.

\section{Trilineage Differentiation and Cell Surface Marker Expression}

MSCs at passage 3 underwent trilineage differentiation into adipocytes, chondrocytes, and osteocytes, and cell surface marker expression of MHCI, MHCII, CD29, CD45, and CD90 was evaluated as previously described $(21,22)$.

\section{Mixed Lymphocyte Reactions}

Previously cryopreserved MSCs were thawed and plated at 50,000 cells per well for $24 \mathrm{~h}$ prior to inactivation with mitomycin C (Sigma Aldrich) as previously described (23). Responder and stimulator lymphocytes were isolated from two unrelated donors using a Ficoll (GE Healthcare) gradient with the addition of carbonyl iron (Sigma Aldrich) (24). Stimulator lymphocytes were inactivated by incubation with $50 \mu \mathrm{g} / \mathrm{ml}$ mitomycin C for $30 \mathrm{~min}$ and then added at a density of $1 \times 10^{6}$ stimulator lymphocytes per well. Responder lymphocytes were stained

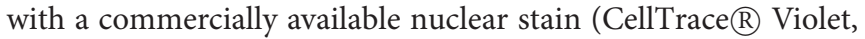
Thermo Fisher) and $2 \times 10^{6}$ responder lymphocytes were added to each well. Cultures were maintained for 5 days, after which lymphocytes were collected and stained with anti-equine CD3+ antibody (UC Davis) at a 1:200 dilution. Flow cytometry was then performed on CD3 $+\mathrm{T}$ lymphocytes to assess proliferation with the use of commercially available software (FlowJo ${ }^{\mathrm{TM}}$ Software). Stained, unstimulated responder lymphocytes were used as a negative proliferation control, Concanavalin A (Sigma Aldrich) stimulated responder lymphocytes were used as a positive proliferation control, and changes in mean fluorescence intensity were evaluated as a percent change from the negative control as previously described (25).

\section{Equine Model}

On day 0, $10 \times 10^{6}$ autologous MSCs prepared respective of group assignment were thawed at $37^{\circ} \mathrm{C}$ and administered to the left metacarpophalangel joint by intra-articular injection in cryopreservation media (95\% autologous serum and 5\% DMSO). On day 29, autologous MSC injection was repeated with $25 \mathrm{ng}$ LPS (lipopolysaccharide from Escherichia coli O55:B5, Sigma Aldrich) suspended in saline and injected immediately prior to MSCs.

\section{Clinical Evaluation}

Physical examinations including heart rate, respiratory rate, and temperature were performed prior to and every $12 \mathrm{~h}$ for 3 days after each injection. As a measurement of pain, gait asymmetry was quantified using an inertial-based sensor system (Lameness Locator, Equinosis $囚$ ) prior to and after each injection (days $0,1,2,3,7,29,30,31,32$, and 36). Peri-articular edema and synovial effusion were scored at the same time points; $0=$ no edema/effusion, $1=$ mild edema/effusion, $2=$ moderate edema/effusion, 3 = severe edema/effusion. Limb circumference was measured at the level of distal metacarpophalangeal IV on days $29,30,31,32$, and 36 .

\section{Synovial Cytology, Cytokine and Chemokine Analysis}

Synovial fluid was collected from the left metacarpophalangeal joint prior to and after each injection on days $0,1,2,3,7$, $29,30,31,32,36$ and examined by a board-certified veterinary pathologist. Any effect of repeated synoviocentesis would have equal chance to affect both groups $(26,27)$. Synovial fluid analysis including total nucleated cell count, cellular differential, and total protein measurement was performed on all samples.

Synovial fluid collected on days 1 and 30 was also analyzed using a 23 analyte, equine specific, multiplex kit (Millipore Sigma) as previously described (28). Analytes measured included: FGF-2, eotaxin, G-CSF, IL-1 $\alpha$, GM-CSF, fractalkine, IL-13, IL-5, IL-18, IL-1 $\beta$, IL-6, IL-17a, IL-2, IL-4, IL-12, IFN $\gamma$, IL-8, IP-10, GRO, MCP-1, IL-10, TNF $\alpha$, and RANTES.

\section{Synovial Colony Forming Unit-Fibroblast Assay}

On days 1, 7, 30, and 36, eight drops of synovial fluid from the $20 \mathrm{~g} 1.5$ inch needle, or $\sim 1 \mathrm{ml}$, were plated to a $10 \mathrm{~cm}$ tissue culture dish along with MSC culture media. Media was changed after $24 \mathrm{~h}$ and again $72 \mathrm{~h}$ later. After 7 days in culture, dishes 
A

\begin{tabular}{lcccccccccccc} 
& CD29 & CD29 & CD44 & CD44 & CD45 & CD45 & CD90 & CD90 & MHCI & MHCI & MHCII & MHCII \\
& BMS & FBS & BMS & FBS & BMS & FBS & BMS & FBS & BMS & FBS & BMS & FBS \\
\hline Horse 1 & $100 \%$ & $99.9 \%$ & $78.0 \%$ & $82.4 \%$ & $1.94 \%$ & $12.7 \%$ & $99.1 \%$ & $90.4 \%$ & $99.8 \%$ & $98.7 \%$ & $1.11 \%$ & $0.59 \%$ \\
Horse 2 & $99.6 \%$ & $100 \%$ & $45.2 \%$ & $43.0 \%$ & $2.81 \%$ & $4.19 \%$ & $99.5 \%$ & $90.0 \%$ & $99.8 \%$ & $100 \%$ & $1.95 \%$ & $1.31 \%$ \\
Horse 3 & $99.9 \%$ & $100 \%$ & $65.4 \%$ & $81.2 \%$ & $1.93 \%$ & $2.26 \%$ & $96.6 \%$ & $95.4 \%$ & $93.5 \%$ & $87.6 \%$ & $3.09 \%$ & $0.82 \%$ \\
Horse 4 & $99.9 \%$ & $100 \%$ & $60.4 \%$ & $70.0 \%$ & $2.65 \%$ & $21.2 \%$ & $99.4 \%$ & $95.7 \%$ & $100 \%$ & $100 \%$ & $1.04 \%$ & $1.68 \%$ \\
Horse 5 & $100 \%$ & $100 \%$ & $62.8 \%$ & $35.6 \%$ & $2.21 \%$ & $13.1 \%$ & $99.7 \%$ & $99.6 \%$ & $99.2 \%$ & $91.2 \%$ & $0.80 \%$ & $1.04 \%$ \\
Horse6 & $99.8 \%$ & $99.9 \%$ & $4.87 \%$ & $7.82 \%$ & $0.62 \%$ & $0.09 \%$ & $97.7 \%$ & $76.6 \%$ & $99.4 \%$ & $98.8 \%$ & $0.03 \%$ & $0.53 \%$
\end{tabular}

B
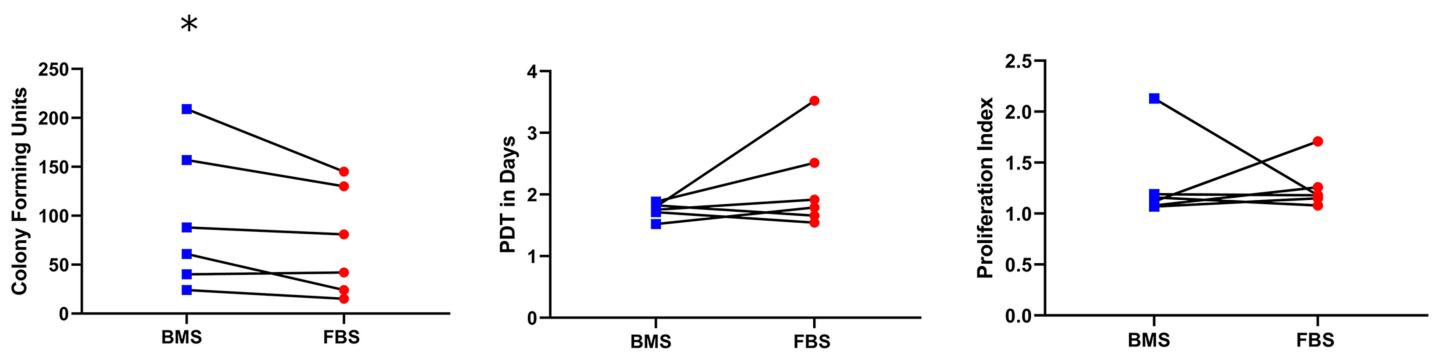

C
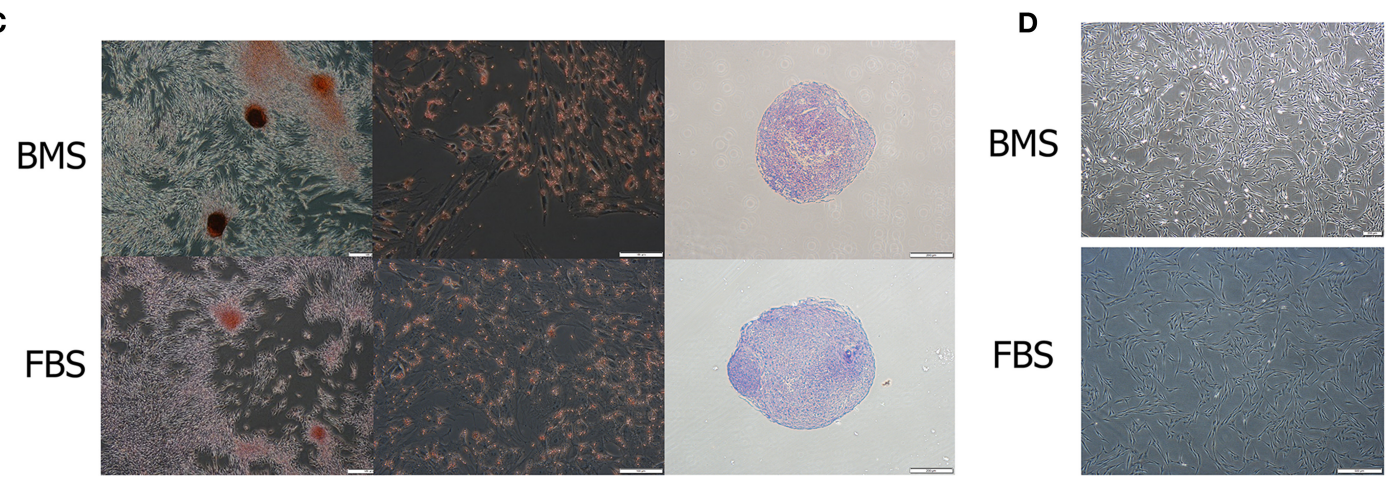

FIGURE 1 | MSC isolation is increased in BMS-MSCs compared to FBS-MSCs; expansion and characterization are not different. (A) Table of percentage of cell surface marker present on MSCs; there was no difference in cell surface marker expression. (B) There was greater isolation of CFU-f colonies with BMS compared to FBS ( $\left.{ }^{*} p \leq 0.05\right)$, but no difference in population doubling time (PDT) from isolation to passage 3 , or immunomodulation, measured by lymphocyte proliferation index in mixed lymphocyte reactions. (C) Trilineage differentiation into bone, fat, and cartilage was not different between BMS-MSCs and FBS-MSCs. (D) BMS-MSCs were similar in appearance to FBS-MSCs.

were stained with $3 \%$ crystal violet, allowed to dry overnight, and colonies counted without magnification.

\section{Anti-FBS Antibody Concentrations}

Blood was collected on all horses weekly prior to injection and for 8 weeks after the first injection (days $0,7,14,21,28,35$, 42,49 , and 56). An anti-FBS antibody ELISA was performed as previously described $(29,30)$. Briefly, plates were coated with FBS from the same lot as MSC preparation, and incubated overnight. Plates were washed, serum was added at a 1:3,200 dilution, and for $30 \mathrm{~min}$. Secondary antibody (Abcam) was added at 1:20,000 dilution for $30 \mathrm{~min}$. After a final wash, $100 \mu \mathrm{l}$ of TMB (Genway Biotech Inc.) was added followed by $100 \mu \mathrm{l}$ of stop solution
(Genway Biotech Inc.) 15 min later. Plates were read at $450 \mathrm{~nm}$, and optical density (OD) reported. Fetal equine serum (FES) was used as a negative assay control, which had the same optical density as the blank control. Titers were then measured by repeating the above ELISA procedure with serial dilutions (from $1: 1,600$ to $1: 819,200)$ of serum collected from the FBS group on days 0 and 56 .

\section{Microcytotoxicity Assay}

Microcytotoxicity assays were performed using serum collected weekly (days $0,7,14,21,28,35,42,49$, and 56) and synovial fluid collected prior to and after the first and second injections (days 0, 1, 7, 30, and 36) with autologous MSCs cultured in 
A

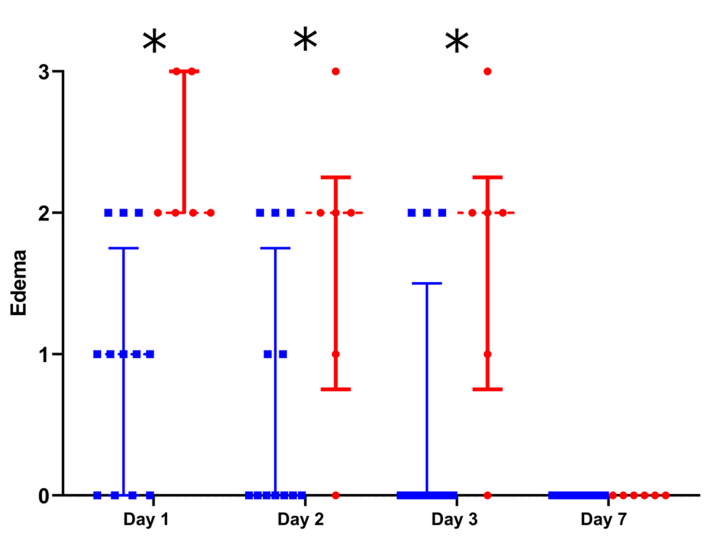

B

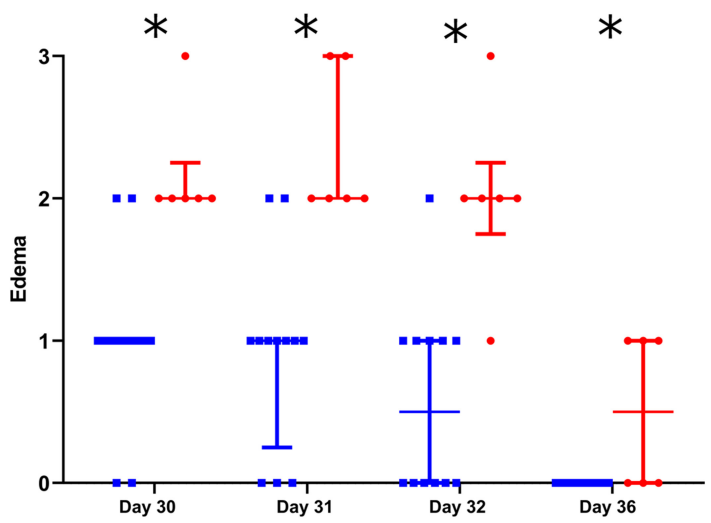

C

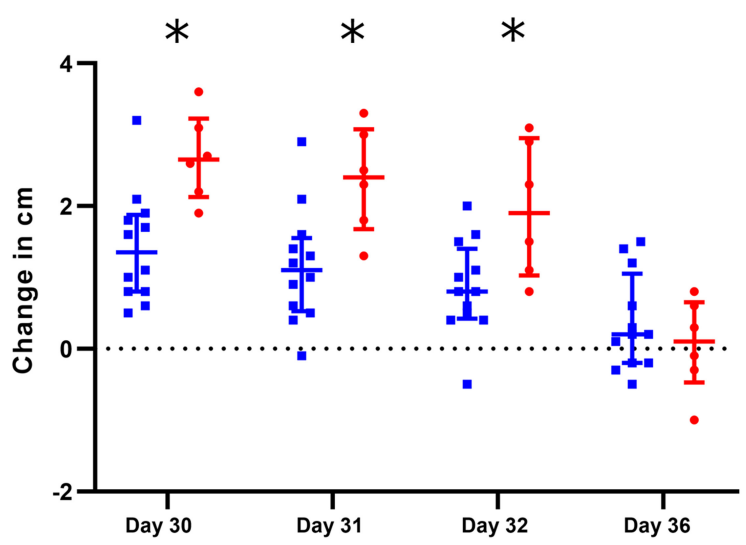

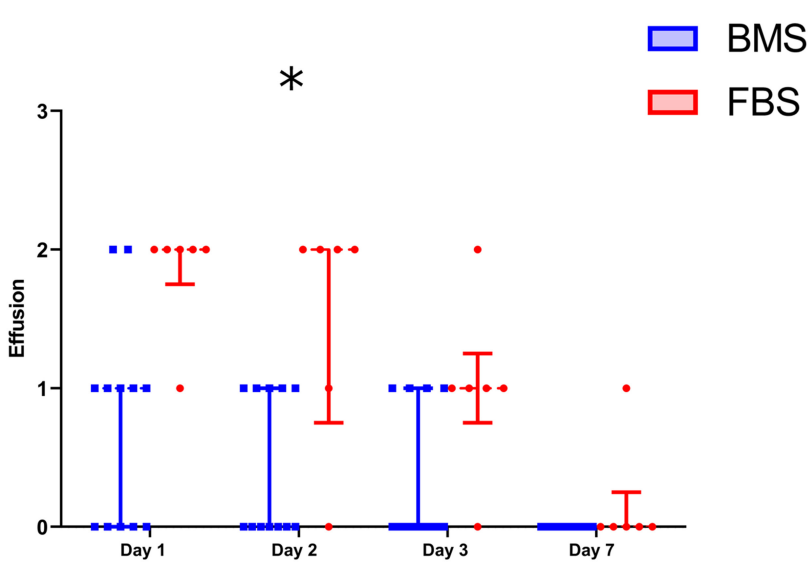

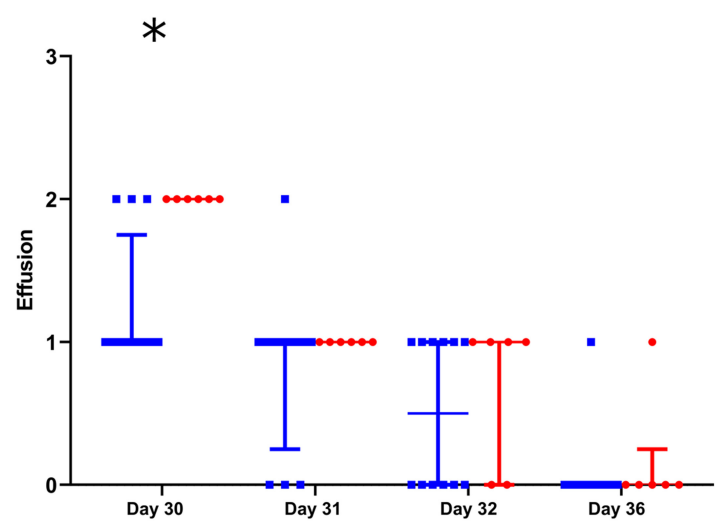

D

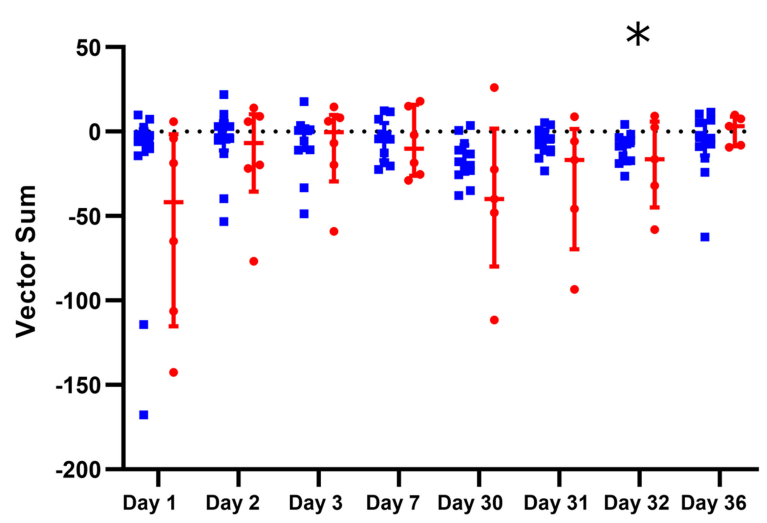

FIGURE 2 | Local adverse response in FBS-MSC recipients compared to BMS-MSC recipients. (A) Peri-articular edema and synovial effusion was worsened in FBS-MSC recipients (significance denoted by asterisks, ${ }^{*} p \leq 0.05$ ). (B) After the second injection, with concurrent administration of LPS, peri-articular edema and synovial effusion was again, worsened in FBS-MSC recipients. (C) Likewise, limb circumference was increased after the second injection in FBS-MSC recipients. (D) There was no difference in gait asymmetry (pain) after the first injection. After the second injection, with concurrent LPS administration, there was a trend of worsened gait asymmetry in FBS-MSC recipients on day 30 and 31, and worsened gait asymmetry on day 32 in FBS-MSC recipients.

either BMS or FBS. Briefly, $2 \mu \mathrm{l}$ of serum or synovial fluid was added to a Terasaki plate and $5 \mu$ l of paraffin oil (Sigma Aldrich) layered on top. Autologous MSCs were suspended in DPBS at a concentration of 1,000 cells $/ \mu 1$ and $2 \mu \mathrm{l}$ of the suspension added to each well, ensuring that the cell solution was in contact with the serum or synovial fluid. After $30 \mathrm{~min}$ at room temperature,
$5 \mu \mathrm{l}$ of rabbit complement was added (One Lambda) and plates were incubated for another $60 \mathrm{~min}$ at room temperature. Two $\mu \mathrm{l}$ of $5 \%$ eosin (Sigma Aldrich) was then added to each well, after $5 \mathrm{~min} 5 \mu \mathrm{l}$ of $10 \%$ formalin (Thermo Scientific) was added. Cells were allowed to settle overnight and percentage of cell death was visually assessed at $20 \mathrm{X}$ magnification within $24 \mathrm{~h}$, in a 


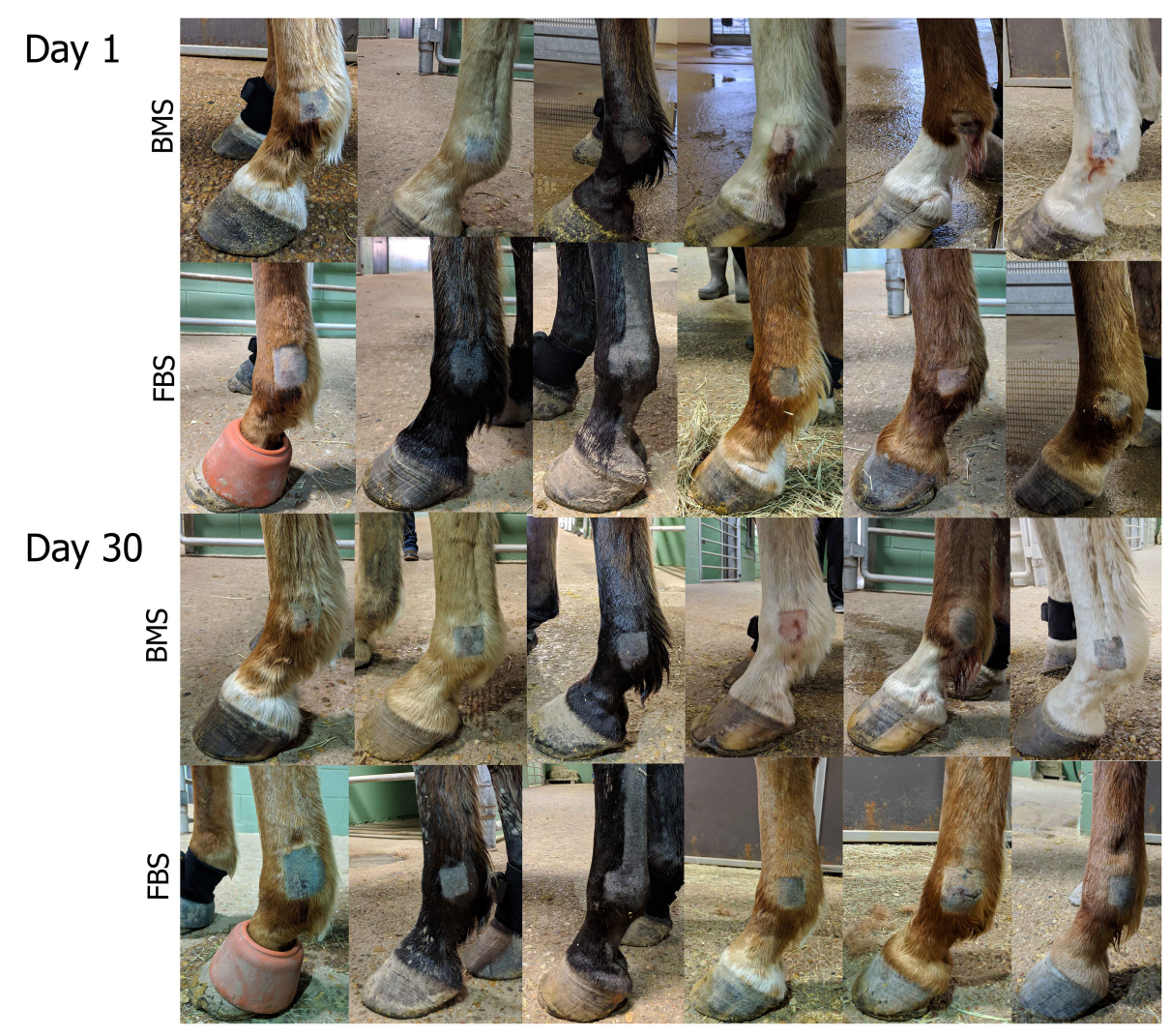

FIGURE 3 | Post-injection edema was marked in FBS-MSC recipients compared to BMS-MSC recipients. Photographs taken on days 1 and 30 , one day after FBS-MSC or BMS-MSC injection. There was worse swelling that retained impression (i.e., pitting edema) in FBS-MSC recipients.

blinded manner as previously described (31). Fetal equine serum was used as a negative control and MHCI specific monoclonal antibody (CZ3.2, provided by Donald Miller), was used as a positive control.

\section{Immunoglobulin Depletion}

To confirm that cell death in the microcytotoxicity assays was anti-FBS antibody mediated, immunoglobulins were depleted from serum collected from FBS-MSC recipients on day 35 as previously described (32). Briefly, a commercially available kit with a Protein A column (ProteoExtract $\AA$, Merck KGaA) was used followed by manual depletion with Sepharose $G$ beads (Millipore Sigma). One hundred $\mu \mathrm{l}$ of serum was diluted in $900 \mu \mathrm{l}$ of $1 \mathrm{x}$ binding buffer. Samples were passed through the Protein A column to remove IgG, in a dropwise manner resulting in partial IgG removal. Two hundred $\mu$ l of preconditioned Sepharose $\mathrm{G}$ beads was added to $300 \mu \mathrm{l}$ of undiluted eluate and incubated for $1 \mathrm{~h}$ with gentle mixing for complete IgG removal. Microcytotoxicity assays were repeated with undiluted serum, serum diluted in $1 \mathrm{x}$ binding buffer, partial immunoglobulin depleted serum, or complete immunoglobulin depleted serum. Assays were completed in duplicate, with donor MSCs cultured in either BMS or FBS.

\section{Statistical Analysis}

Differences in in vitro data between groups were evaluated by paired Wilcoxon signed rank. In vivo, edema, effusion, limb circumference, and lameness were normalized to baseline (day 0 and day 29) prior to MSC injection. Differences between groups and over time in edema, effusion, limb circumference, lameness, and number of colonies present were tested using Kruskal Wallis or Wilcoxon rank sum tests. As a follow-up, a mixed model was used for all in vivo data, no differences were found between either analysis. Differences in proportion of joints positive for MSC colonies were tested using Fisher's exact test at each time point. Groups were considered different when the $p<0.05$.

\section{RESULTS}

\section{Greater MSC Isolation With BMS, but no Difference in Expansion, Characterization, or Immunomodulatory Capacity in BMS-MSCs Compared to FBS-MSCs}

First, we investigated whether BMS supports MSC isolation and expansion without differences to MSC characterization or immunomodulatory capacity compared to FBS supplementation. There was an increased rate of MSC colony isolation after 

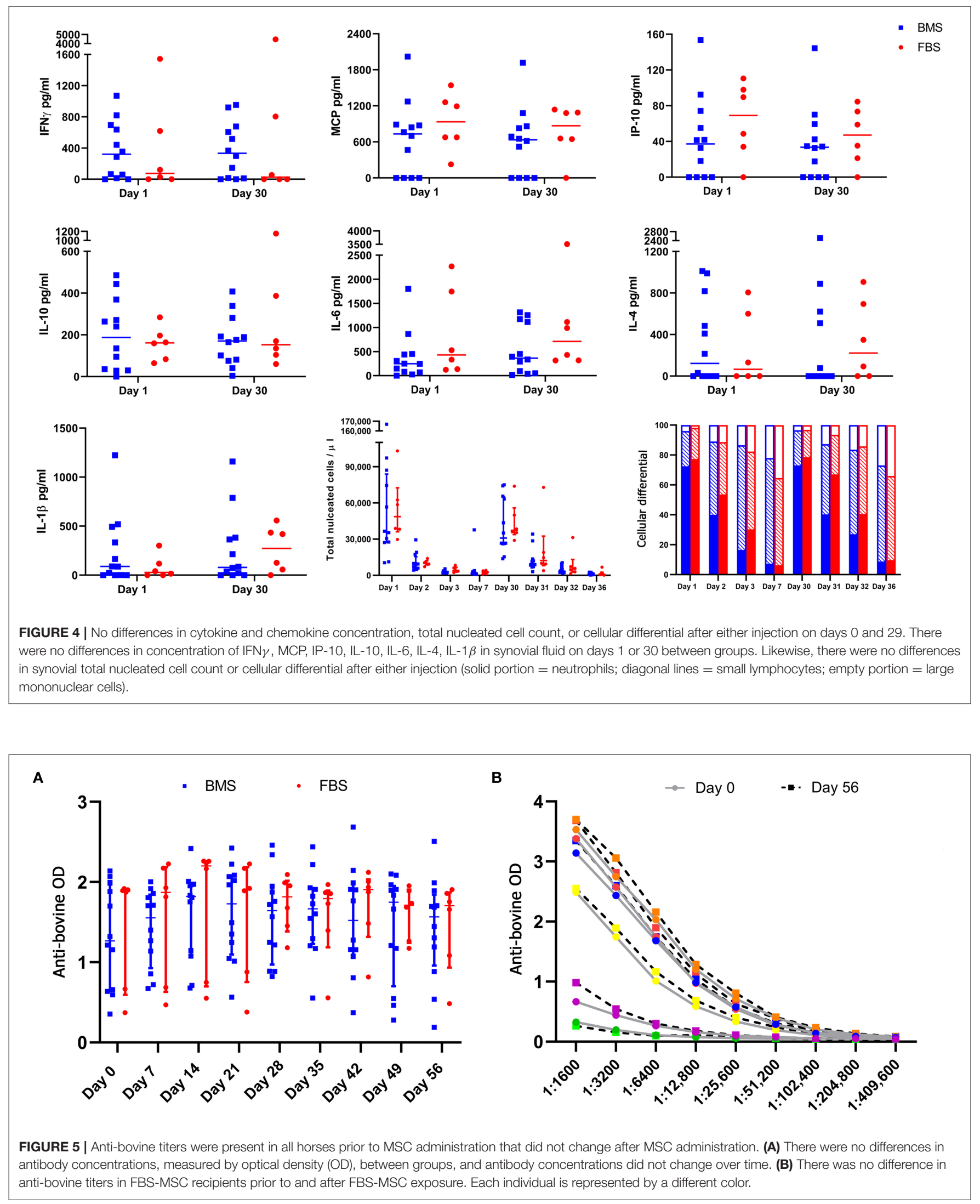

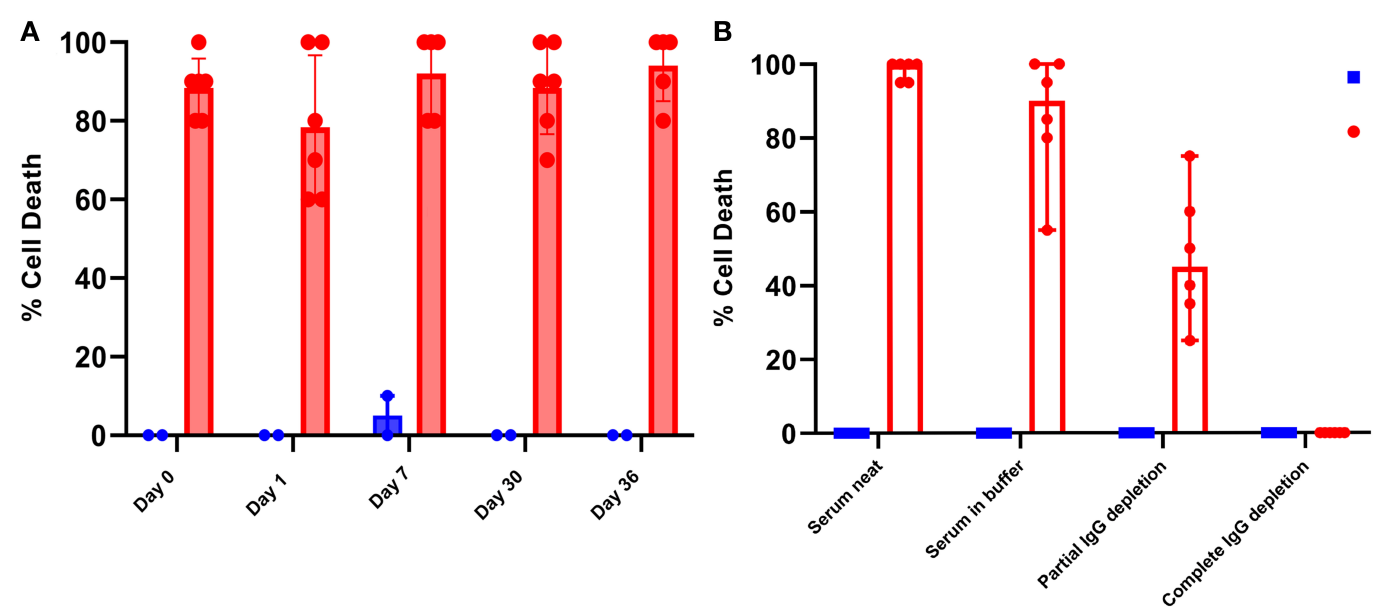

FIGURE 6 | Antibodies against bovine proteins are present in serum and synovial fluid, and cause death of FBS prepared MSCs. (A) Synovial fluid collected on days $0,1,7,30$, and 36 produced significant cell death when combined with autologous FBS-MSCs. (B) To demonstrate that MSC cell death was antibody mediated, serum collected on day 35 was depleted of immunoglobulins partially and completely. All serum samples were combined with either BMS-MSCs or FBS-MSCs. There was no death of BMS-MSCs, there was significant cell death of FBS-MSCs both neat and in buffer, a reduction of cell death with partial IgG depletion, and elimination of cell death with complete IgG depletion.

BMS supplementation compared to FBS, but no differences in expansion rate between BMS-MSCs and FBS-MSCs (Figure 1).

No differences in immunomodulatory function were seen using modified one-way mixed lyphocyte reactions (Figure 1). After three passages, BMS-MSCs and FBS-MSCs were phenotypically similar without appreciable differences in morphology, and there were no differences in cell surface marker expression or trilineage differentiation into bone, cartilage, and fat (Figure 1).

\section{FBS-MSCs, but Not BMS-MSCs, Cause Local Inflammation and Are Targeted by the Recipient Immune System}

Eighteen horses received intra-articular injection of autologous MSCs prepared with media supplemented with autologous BMS $(n=6)$, pooled BMS $(n=6)$, or FBS $(n=6)$. Intra-articular injections occurred on experimental day 0 and 29. There were no differences between the autologous and pooled BMS-prepared groups in clinical or laboratory findings including lameness, synovial CFU-f, edema, effusion, cytokine and chemokine concentrations, or cytotoxicity of MSCs; therefore, pooled and autologous BMS data were combined to a single group (BMSMSC, $n=12)$ and compared to FBS $(n=6)$.

\section{FBS Contamination Causes Local Inflammation and Adverse Clinical Response}

After each intra-articular injection, there was increased periarticular edema and synovial effusion in FBS-MSC recipients compared to BMS-MSC recipients (Figures 2, 3) (33, 34). There were no differences in pain between groups after the first injection. One FBS-MSC recipient was removed from gait analysis for assessment of pain after the second injection because of a right forelimb lameness, not related to the study. After the second MSC injection, when LPS was also administered, there was reduced MSC efficacy with a trend of more pain in FBSMSC recipients on day 30 and 31, and significantly worse pain in FBS-MSC recipients on day 32 (Figure 2) $(9,18)$.

\section{No Difference in Synovial Fluid Cytology, Cytokine or Chemokine Concentrations}

There were no differences in synovial fluid cytology (total nucleated cell count or proportion of cell type) after either injection (Figure 4). Synovial cytokines and chemokines revealed measurable concentrations of IFN $\gamma$, MCP, IP-10, IL-10, IL-6, IL4 , and IL- $1 \beta$, but no differences between groups the day after each injection (Figure 4). This single time point may have missed differences due to LPS, but were the same time points as our recent report of significant synovial differences due to allogeneic MHCI mismatch (28).

\section{Anti-bovine Antibodies Were Present and Unchanged in all Horses}

Anti-bovine antibody concentrations were not different between groups or over time (Figure 5). Titers varied by individual, with a median maximum titer of 1:204,800 (range, 1:12,800-1:409,600) without differences in anti-bovine antibody titers between day 0 and 56 (Figure 5).

\section{Anti-bovine Antibodies in the Recipient Cause Death of FBS-MSCs, but Not BMS-MSCs}

While it is well documented that anti-bovine antibodies are present in human and equine serum, little is known about the consequence of these antibodies relative to FBS-MSCs $(35,36)$. It has been considered that the immune modulating properties of MSCs could prevent recipient immune recognition of bovine proteins from FBS that are presented by MHCI as part of normal cellular surveillance (37). Microcytotoxicity assays with recipient 


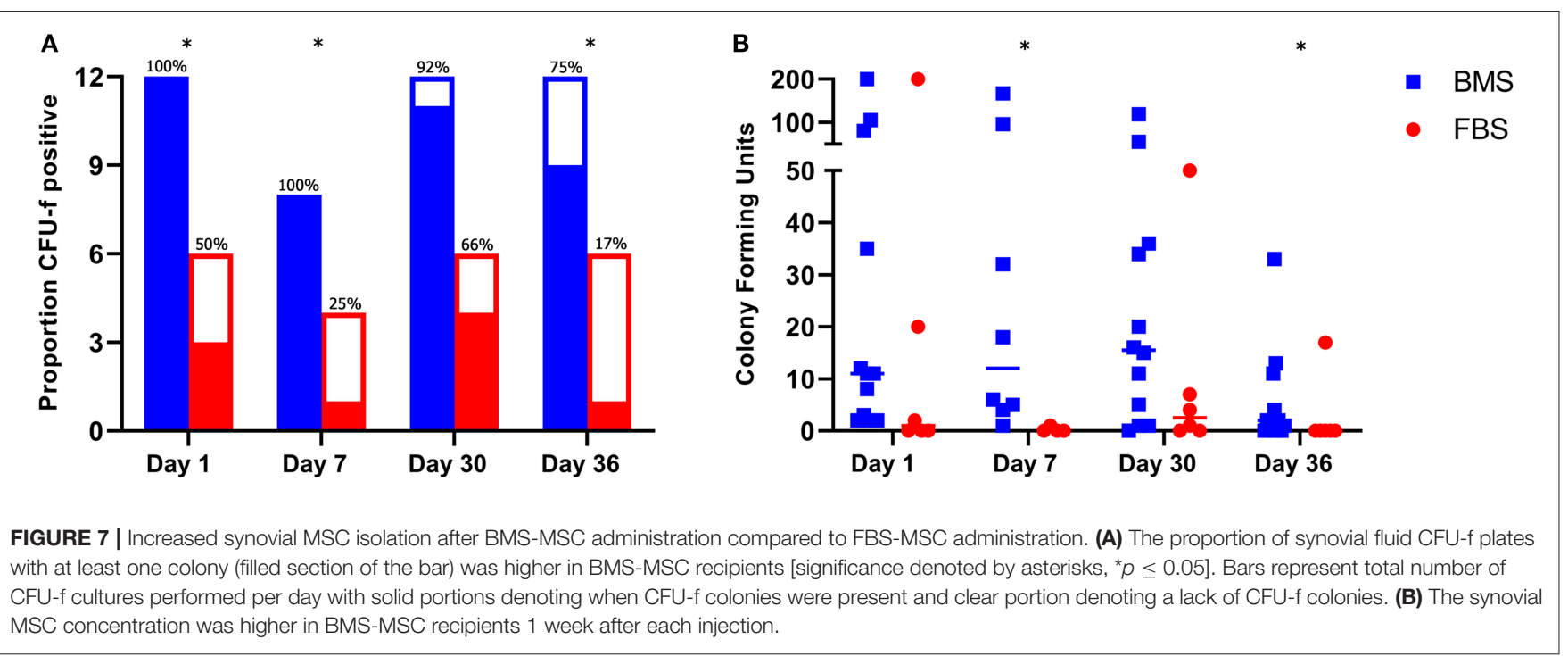

serum and autologous MSCs resulted in widespread death of FBS prepared MSCs. In contrast, there was virtually no cytotoxicity of BMS-MSCs (Figure 6).

To confirm that death of FBS-MSCs in the microcytotoxicity assay was due to antibody, we repeated mircrocytoxocity assays with partially and fully immunoglobulin depleted serum. There was virtually no cell death of BMS-MSCs when combined with serum in buffer, partial, or complete immunoglobulin depleted serum. In contrast, there was marked death of FBS-MSCs when combined with serum in buffer, a reduction in cell death with partial immunoglobulin depletion, and absence of cell death after complete immunoglobulin depletion (Figure 6).

\section{Pre-existing Anti-bovine Antibodies in Synovial Fluid Cause FBS-MSC Death}

After demonstrating the presence of consistent and unchanged anti-bovine antibodies in serum capable of causing death of FBS-prepared MSCs, we wanted to evaluate if antibodies are of consequence in the articular environment. This is of particular importance because the articular environment is often considered to be immune privileged, as it is nearly acellular with a distinct blood-joint barrier that minimizes diffusion of small molecules $(38,39)$. We repeated microcytotoxicity assays combining FBS-MSCs or BMS-MSCs with synovial fluid collected on days $0,1,7,30$, and 36 after intra-articular MSC administration. At all time-points, there was cytotoxic FBS-MSC death but not BMS-MSC, confirming that anti-FBS antibodies are present in synovial fluid in sufficient quantities to cause cytotoxic cell death of FBS contaminated MSCs before and after intra-articular injections (Figure 6).

\section{Joints Injected With FBS-MSCs Have Lower Synovial MSC Concentrations Compared to Those Injected With BMS-MSCs}

To assess for differences in MSC survival within the joint, we measured synovial MSC concentrations using CFU-f assays on days 1, 7, 30, and 36; the measured MSCs may have been either endogenous progenitors within synovial fluid or injected MSCs that had survived. The proportion of CFU-f plates with at least one colony was higher on days 1, 7, and 36 in BMS-MSC recipients compared to FBS-MSC recipients (Figure 7). The total number of colonies from each joint was higher 1 week after each injection on days 7 and 36 in BMS-MSC recipients compared to FBS-MSC recipients (Figure 7).

\section{DISCUSSION}

We demonstrate that the recipient immune response to FBS prepared autologous MSCs results in MSC death, local inflammation, and reduced synovial MSC concentrations. Recent failures of therapeutic MSCs to achieve market approval in the United States, despite foreign regulatory approval, might be due to FBS use during MSC preparation resulting in altered clinical effect and failure to meet stringent end points $(4,8,9,35,40)$. While clinical human MSC preparation is transitioning away from FBS use, continued FBS supplementation of MSCs in animal models and veterinary clinical application will obfuscate clinical translation $(9,34,41)$.

Our findings refute the conclusion that anti-bovine titers are not of consequence because they do not change with repeated exposure to FBS-MSCs $(11,14,17)$. We suggest the lack of change in anti-bovine titers, described by others and here, is because peak titers that are incapable of an anamnestic response exist prior to MSC therapy $(11,17)$. In horses, these peak titers are due to routine bi-annual vaccination against viral pathogens, which are also prepared with FBS (36). This frequent vaccination results in inadvertent, but thorough, vaccine induced immunity and peak titers against bovine proteins (36).

Veterinary reports have attributed inflammatory events after MSC therapy to a normal physiologic response to MSCs, and in people immunomodulatory drugs are commonly administered to mitigate inflammation during MSC administration (9, 17, 34, 4244). We show that adverse reactions and local inflammation 
are not the normal physiologic response to MSCs, rather a consequence of FBS contamination of MSCs $(33,34)$. Moreover, the articular environment that we used is particularly well suited to investigate these adverse events because the blood-joint barrier and large volume-to-surface area ratio sequesters the local response, augmenting detection of inflammation $(38,39)$. Given this exquisite sensitivity of the articular joint to inflammation, the absence of edema or effusion in BMS-MSC recipients without concurrent anti-inflammatory administration or limb wrapping support is remarkable.

In a recent report from our group using the same experimental model, we showed differences in synovial cytokine concentrations, including IFN $\gamma, 1$ day after mismatched allogeneic MSCs were administered compared to matched allogeneic MSCs (28). Intriguingly, in the current report, we did not see differences in synovial cytokines at this same time point, despite marked synovial effusion and peri-articular edema that lasted several days in FBS-MSC recipients. This difference in degree and duration of inflammation, with greater degree of inflammation in mismatched recipients (higher IFN-gamma on days 1 and 30) reported previously and longer duration of inflammation in FBS-MSC recipients (more peri-articular edema for the entire week after each intra-articular injection) reported here, is likely because of differences in the mechanism of antigen recognition. With allogeneic mismatch, MHCI incompatibility would result in every MSC being immediately identified as foreign. Whereas, FBS prepared MSCs presenting cellular contents in routine cell monitoring may not initially be presenting bovine antigen on their MHCI, may be presenting very little bovine antigen on their MHCI, or may not present bovine antigen on their MHCI until days after administration. It is also possible that the prolonged edema and effusion in the FBS-MSC group is due to exocytosis of bovine antigen by FBS prepared MSCs or by recognition of bovine antigen by IgE, which is often present in horses (36).

We also recently reported increased endogenous progenitors in joints injected with MHCI matched allogeneic MSCs compared to mismatched allogeneic MSCs (28). In the report here, all injected MSCs were autologous and whether the increased synovial MSC concentration after BMS-MSC administration was due to improved MSC persistence or endogenous progenitor upregulation cannot be distinguished. Nonetheless, recipient immune targeting of FBS-MSCs resulted in lower synovial MSC concentrations.

Continued acceptance of FBS for veterinary and pre-clinical study may be due to the marked failures of platelet products to adequately support FBS-free preparation of animal derived MSCs, the high cost of chemically define media, and the wellknown failure of adult derived serum to support MSC isolation and expansion (45-48). After years of failure by our group to develop equine platelet lysate or releasate for isolation and longterm expansion without alteration of MSC characteristics (data not shown), we demonstrated that replacement of FBS with autologous serum for $48 \mathrm{~h}$ greatly reduces FBS contamination of equine MSCs, but does not eliminate it (16). In our efforts to eliminate FBS contamination, we discovered BMS as an FBS alternative. Advantages of BMS is that it can be autologous, is inexpensive to obtain and process, and is a by-product when utilizing bone marrow derived MSCs. A disadvantage is that large volumes of bone marrow are required to support isolation and expansion for the entire MSC culture duration.

Given the unchanged growth and metabolism of BMS-MSCs as compared to FBS-MSCs, it is possible that BMS in the final stages of MSC preparation could be used to more thoroughly eliminate FBS contamination as compared to adult derived serum (16). Additionally, we do not know which factor, or factors, is present in BMS and not in adult derived serum. In the absence of this knowledge, we assessed for BMS quality differences by comparing to FBS in vitro and by comparing autologous and pooled BMS in vivo, which did not have differences. This suggests that BMS quality was consistent in this group of horses, but further work is needed.

We show that recipient anti-bovine titers cause antibody mediated death of MSCs that have been prepared with FBS, with resultant local inflammation and reduced synovial MSCs after intra-articular administration. The historic and current use of FBS for MSC preparation is likely to misrepresent MSC effect because of cytotoxicity and adverse responses to MSCs with FBS contamination (10, 42, 49). When evaluating reported pre-clinical, veterinary, and human clinical trials, the use of FBS should be considered when interpreting results (4, 9, 40, 41). Bone marrow supernatant is a simple, inexpensive, and autologous replacement for FBS that eliminates immune targeting and the resultant adverse clinical effects. Fetal bovine serum should not be used for MSC supplementation in veterinary, pre-clinical or clinical MSC preparation, and the use of BMS should be further investigated.

\section{DATA AVAILABILITY STATEMENT}

The raw data supporting the conclusions of this article will be made available by the authors, without undue reservation.

\section{ETHICS STATEMENT}

The animal study was reviewed and approved by Texas A\&M University Institutional Animal Care and Use Committee.

\section{AUTHOR CONTRIBUTIONS}

AR and GL: study design, data acquisition, analysis and interpretation of data, and manuscript preparation. MB: data acquisition, analysis and interpretation of data, and manuscript preparation. AW: study design, analysis, data acquisition and interpretation of data, and manuscript preparation. All authors contributed to the article and approved the submitted version. 


\section{FUNDING}

Funding was provided by the Link Endowment for Equine Research at Texas A\&M University, and the Linda and Dennis H. Clark '68 Endowed Chair for Equine Studies.

\section{REFERENCES}

1. Barry F. MSC therapy for osteoarthritis: an unfinished story. J Orthop Res. (2019) 37:1229-35. doi: 10.1002/jor.24343

2. Galipeau J, Sensébé L. Mesenchymal stromal cells: clinical challenges and therapeutic opportunities. Cell Stem Cell. (2018) 22:824-33. doi: 10.1016/j.stem.2018.05.004

3. Trounson A, McDonald C. Stem cell therapies in clinical trials: progress and challenges. Cell Stem Cell. (2015) 17:11-22. doi: 10.1016/j.stem.2015.06.007

4. Levy O, Kuai R, Siren EMJ, Bhere D, Milton Y, Nissar N, et al. Shattering barriers toward clinically meaningful MSC therapies. Sci Adv. (2020) 6:eaba6884. doi: 10.1126/sciadv.aba6884

5. Pittenger MF, Discher DE, Péault BM, Phinney DG, Hare JM, Caplan AI. Mesenchymal stem cell perspective: cell biology to clinical progress. NPJ Regen Med. (2019) 4:22. doi: 10.1038/s41536-019-0083-6

6. Friedenstein AJ, Chailakhyan RK, Latsinik NV, Panasyuk AF, Keiliss-Borok IV. Stromal cells responsible for transferring the microenvironment of the hemopoietic tissues. Cloning in vitro and retransplantation in vivo. Transplantation. (1974) 17:331-40. doi: 10.1097/00007890-197404000-00001

7. Karnieli O, Friedner OM, Allickson JG, Zhang N, Jung S, Fiorentini $\mathrm{D}$, et al. A consensus introduction to serum replacements and serum-free media for cellular therapies. Cytotherapy. (2017) 19:155-69. doi: 10.1016/j.jcyt.2016.11.011

8. Wang K, Jiang Z, Webster KA, Chen J, Hu H, Zhou Y, et al. Enhanced cardioprotection by human endometrium mesenchymal stem cells driven by exosomal microRNA-21. Stem Cells Transl Med. (2017) 6:209-22. https://doi. org/10.5966/sctm.2015-0386.

9. Panés J, García-Olmo D, Van Assche G, Colombel JF, Reinisch W, Baumgart DC, et al. Expanded allogeneic adipose-derived mesenchymal stem cells $(\mathrm{Cx} 601)$ for complex perianal fistulas in Crohn's disease: a phase 3 randomised, double-blind controlled trial. Lancet. (2016) 388:128190. doi: 10.1016/S0140-6736(16)31203-X

10. Colbath AC, Dow SW, Hopkins LS, Phillips JN, McIlwraith CW, Goodrich LR. Single and repeated intra-articular injections in the tarsocrural joint with allogeneic and autologous equine bone marrow-derived mesenchymal stem cells are safe, but did not reduce acute inflammation in an experimental interleukin-1 $\beta$ model of synovitis. Equine Vet J. (2020) 52:60112. doi: $10.1111 /$ evj.13222

11. Owens SD, Kol A, Walker NJ, Borjesson DL. Allogeneic mesenchymal stem cell treatment induces specific alloantibodies in horses. Stem Cells Int. (2016) 2016:5830103. doi: 10.1155/2016/58 30103

12. Selvaggi TA, Walker RE, Fleisher TA. Development of antibodies to fetal calf serum with arthus-like reactions in human immunodeficiency virus-infected patients given syngeneic lymphocyte infusions. Blood. (1997) 89:776-9.

13. Macy E, Bulpitt K, Champlin RE, Saxon A. Anaphylaxis to infusion of autologous bone marrow: an apparent reaction to self, mediated by $\operatorname{IgE}$ antibody to bovine serum albumin. J Allergy Clin Immunol. (1989) 83:8715. doi: 10.1016/0091-6749(89)90099-7

14. Sundin M, Ringdén O, Sundberg B, Nava S, Götherström C, Le Blanc K. No alloantibodies against mesenchymal stromal cells, but presence of anti-fetal calf serum antibodies, after transplantation in allogeneic hematopoietic stem cell recipients. Haematologica. (2007) 92:120815. doi: 10.3324/haematol.11446

15. Spees JL, Gregory CA, Singh H, Tucker HA, Peister A, Lynch PJ, et al. Internalized antigens must be removed to prepare hypoimmunogenic mesenchymal stem cells for cell and gene therapy. Mol Ther. (2004) 9:74756. doi: 10.1016/j.ymthe.2004.02.012

\section{ACKNOWLEDGMENTS}

We would like to thank Carl Gregory, Katrin Hinrichs, Michael Criscitiello, Donald Miller, and Douglas Antczak for their advice throughout this experiment and during manuscript preparation. We would like to also thank Hsing Fann for her technical support.

16. Joswig AJ, Mitchell A, Cummings KJ, Levine GJ, Gregory CA, Smith R, et al Repeated intra-articular injection of allogeneic mesenchymal stem cells causes an adverse response compared to autologous cells in the equine model. Stem Cell Res Ther. (2017) 8:42-8. doi: 10.1186/s13287-017-0503-8 [doi].

17. Arzi B, Mills-Ko E, Verstraete FJM, Kol A, Walker NJ, Badgley MR, et al. Therapeutic efficacy of fresh, autologous mesenchymal stem cells for severe refractory gingivostomatitis in cats. Stem Cells Transl Med. (2016) 5:75-86. doi: 10.5966/sctm.2015-0127

18. Horwitz EM, Gordon PL, Koo WKK, Marx JC, Neel MD, McNall RY, et al. Isolated allogeneic bone marrow-derived mesenchymal cells engraft and stimulate growth in children with osteogenesis imperfecta: implications for cell therapy of bone. Proc Natl Acad Sci U S A. (2002) 99:89327. doi: 10.1073/pnas.132252399

19. Galipeau J. The mesenchymal stromal cells dilemma-does a negative phase III trial of random donor mesenchymal stromal cells in steroid-resistant graftversus-host disease represent a death knell or a bump in the road? Cytotherapy. (2013) 15:2-8. doi: 10.1016/j.jcyt.2012.10.002

20. Rowland AL, Navas de. Solis C, Lepiz MA, Cummings KJ, Watts AE. Bone Marrow Aspiration Does Not Induce a Measurable Pain Response Compared to Sham Procedure. Front. Vet. Sci. (2018) 5:233. doi: 10.3389/fvets.2018.00233

21. Mitchell A, Rivas KA, Smith R, Watts AE. Cryopreservation of equine mesenchymal stem cells in 95\% autologous serum and 5\% DMSO does not alter post-thaw growth or morphology in vitro compared to fetal bovine serum or allogeneic serum at 20 or $95 \%$ and DMSO at 10 or 5. Stem Cell Res Ther. (2015) 6:231-y. doi: 10.1186/s13287-015-0230-y

22. Rowland AL, Xu JJ, Joswig AJ, Gregory CA, Antczak DF, Cummings KJ, et al. In vitro MSC function is related to clinical reaction in vivo. Stem Cell Res Ther. (2018) 9:295. doi: 10.1186/s13287-018-1037-4

23. Paterson YZ, Rash N, Garvican ER, Paillot R, Guest DJ. Equine mesenchymal stromal cells and embryo-derived stem cells are immune privileged in vitro. Stem Cell Res Ther. (2014) 5:90. doi: 10.1186/scrt479

24. Flaminio MJ, Rush BR, Davis EG, Hennessy K, Shuman W, Wilkerson MJ. Characterization of peripheral blood and pulmonary leukocyte function in healthy foals. Vet Immunol Immunopathol. (2000) 73:267-85. doi: 10.1016/s0165-2427(00)00149-5

25. Schnabel LV, Pezzanite LM, Antczak DF, Felippe MJ, Fortier LA. Equine bone marrow-derived mesenchymal stromal cells are heterogeneous in MHC class II expression and capable of inciting an immune response in vitro. Stem Cell Res Ther. (2014) 5:13. doi: 10.1186/scrt402

26. Hunt CL, Leatherwood JL, Coverdale JA, Sigler DL, Vogelsang MM, Arnold CE. Effects of repeated arthrocentesis on systemic cytokine expression and leukocyte population in young horses challenged with intra-articular lipopolysaccharide. J Anim Sci. (2019) 97:184-91. doi: 10.1093/jas/sky423

27. Rinnovati R, Bonelli F, Tognetti R, Gallo C, Bassini RF, Marchetti V, et al. Effect of repeated arthrocentesis on cytology of synovial fluid. J Equine Vet Sci. (2017) 57:112-5. doi: 10.1016/j.jevs.2017.07.008

28. Rowland AL, Miller D, Berglund A, Schnabel LV, Levine GJ, Antczak DF, et al. Cross-matching of allogeneic mesenchymal stromal cells eliminates recipient immune targeting. Stem Cells Transl Med. (2020) 10:694710. doi: 10.1002/sctm.20-0435

29. Gregory CA, Reyes E, Whitney MJ, Spees JL. Enhanced engraftment of mesenchymal stem cells in a cutaneous wound model by culture in allogenic species-specific serum and administration in fibrin constructs. Stem Cells. (2006) 24:2232-43. doi: 10.1634/stemcells.2005-0612

30. Mackensen A, Dräger R, Schlesier M, Mertelsmann R, Lindemann A. Presence of IgE antibodies to bovine serum albumin in a patient developing anaphylaxis after vaccination with human peptide-pulsed dendritic cells. 
Cancer Immunol Immunother. (2000) 49:152-6. doi: 10.1007/s0026200 50614

31. Berglund AK, Schnabel LV. Allogeneic major histocompatibility complexmismatched equine bone marrow-derived mesenchymal stem cells are targeted for death by cytotoxic anti-major histocompatibility complex antibodies. Equine Vet J. (2017) 49:539-44. doi: 10.1111/evj.12 647

32. Olver CS, Webb TL, Long LJ, Scherman H, Prenni JE. Comparison of methods for depletion of albumin and IgG from equine serum. Vet Clin Pathol. (2010) 39:337-45. doi: 10.1111/j.1939-165X.2010.00241.x

33. Barrachina L, Remacha AR, Romero A, Vitoria A, Albareda J, Prades $\mathrm{M}$, et al. Assessment of effectiveness and safety of repeat administration of proinflammatory primed allogeneic mesenchymal stem cells in an equine model of chemically induced osteoarthritis. BMC Vet Res. (2018) 14:241. doi: 10.1186/s12917-018-1556-3

34. Colbath AC, Dow SW, Hopkins LS, Phillips JN, McIlwraith CW, Goodrich LR. Allogeneic vs. autologous intra-articular mesenchymal stem cell injection within normal horses: Clinical and cytological comparisons suggest safety. Equine Vet J. (2019) 52:144-51. doi: 10.1111/evj.13136

35. Andersen DC, Koch C, Jensen CH, Skjødt K, Brandt J, Teisner B. High prevalence of human anti-bovine IgG antibodies as the major cause of false positive reactions in two-site immunoassays based on monoclonal antibodies. J Immunoassay Immunochem. (2004) 25:17-30. doi: 10.1081/ias-120027223

36. Gershwin LJ, Netherwood KA, Norris MS, Behrens NE, Shao MX. Equine IgE responses to non-viral vaccine components. Vaccine. (2012) 30:761520. doi: 10.1016/j.vaccine.2012.10.029

37. Owens SD, Kol A, Borjesson DL. Allogeneic mesenchymal stem cell treatment induces specific alloantibodies in horses. (2016) 2016:5830103.

38. Simkin PA. Synovial permeability in rheumatoid arthritis. Arthritis Rheum. (1979) 22:689-96. doi: 10.1002/art.1780220701

39. Levick JR. Permeability of rheumatoid and normal human synovium to specific plasma proteins. Arthritis Rheum. (1981) 24:1550-60. doi: 10.1002/art.1780241215

40. Oh K, Moon C, Kim HY, Oh S, Park J, Lee JH, et al. Phase I trial of repeated intrathecal autologous bone marrow-derived mesenchymal stromal cells in amyotrophic lateral sclerosis. Stem Cells Transl Med. (2015) 4:5907. doi: 10.5966/sctm.2014-0212

41. Murphy JM, Fink DJ, Hunziker EB, Barry FP. Stem cell therapy in a caprine model of osteoarthritis. Arthritis Rheum. (2003) 48:346474. doi: 10.1002/art.11365

42. Barrachina L, Cequier A, Romero A, Vitoria A, Zaragoza P, Vázquez FJ, et al. Allo-antibody production after intraarticular administration of mesenchymal stem cells (MSCs) in an equine osteoarthritis model: effect of repeated administration, MSC inflammatory stimulation, and equine leukocyte antigen (ELA) compatibility. Stem Cell Res Ther. (2020) 11:52. doi: 10.1186/s13287-020-1571-8
43. Carrade DD, Owens SD, Galuppo LD, Vidal MA, Ferraro GL, Librach F, et al. Clinicopathologic findings following intra-articular injection of autologous and allogeneic placentally derived equine mesenchymal stem cells in horses. Cytotherapy. (2011) 13:419-30. doi: 10.3109/14653249.2010.536213.

44. Quimby JM, Webb TL, Habenicht LM, Dow SW. Safety and efficacy of intravenous infusion of allogeneic cryopreserved mesenchymal stem cells for treatment of chronic kidney disease in cats: results of three sequential pilot studies. Stem Cell Res Ther. (2013) 4:48. doi: 10.1186/scrt198

45. Chapman H, Gale AL, Dodson ME, Linardi RL, Ortved KF. Autologous platelet lysate does not enhance chondrogenic differentiation of equine bone marrow-derived mesenchymal stromal cells despite increased TGF- $\beta 1$ concentration. Stem Cells Dev. (2020) 29:144-55. doi: 10.1089/scd.2019.0239

46. Naskou MC, Sumner S, Berezny A, Copland IB, Peroni JF. Fibrinogen-depleted equine platelet lysate affects the characteristics and functionality of mesenchymal stem cells. Stem Cells Dev. (2019) 28:1572-80. doi: 10.1089/scd.2019.0070

47. Russell KA, Koch TG. Equine platelet lysate as an alternative to fetal bovine serum in equine mesenchymal stromal cell culture - too much of a good thing? Equine Vet J. (2016) 48:261-4. doi: 10.1111/evj.12440

48. Longhini ALF, Salazar TE, Vieira C, Trinh T, Duan Y, Pay LM, et al. Peripheral blood-derived mesenchymal stem cells demonstrate immunomodulatory potential for therapeutic use in horses. PLoS ONE. (2019) 14:e0212642. doi: 10.1371/journal.pone.0212642

49. Ferris DJ, Frisbie DD, Kisiday JD, McIlwraith CW, Hague BA, Major MD, et al. Clinical outcome after intra-articular administration of bone marrow derived mesenchymal stem cells in 33 horses with stifle injury. Vet Surg. (2014) 43:255-65. doi: 10.1111/j.1532-950X.2014.12100.x

Conflict of Interest: The authors declare that the research was conducted in the absence of any commercial or financial relationships that could be construed as a potential conflict of interest.

Publisher's Note: All claims expressed in this article are solely those of the authors and do not necessarily represent those of their affiliated organizations, or those of the publisher, the editors and the reviewers. Any product that may be evaluated in this article, or claim that may be made by its manufacturer, is not guaranteed or endorsed by the publisher.

Copyright (C) 2021 Rowland, Burns, Levine and Watts. This is an open-access article distributed under the terms of the Creative Commons Attribution License (CC BY). The use, distribution or reproduction in other forums is permitted, provided the original author(s) and the copyright owner(s) are credited and that the original publication in this journal is cited, in accordance with accepted academic practice. No use, distribution or reproduction is permitted which does not comply with these terms. 